\title{
LIMIARES DE TEMPERATURA DE SUPERFÍCIE E PLUVIOSIDADE COMO INDICADOR DE RISCO DE PROLIFERAÇÃO DE Aedes aegypti NO MUNICÍPIO DE SÃO PAULO
}

\author{
Suely Franco Siquera de Lima \\ Universidade de São Paulo Faculdade de Filosofia, Letras e Ciências Humanas Departamento de Geografia, São \\ Paulo, SP, Brasil \\ suelyfrancosiqueira@gmail.com \\ Ligia Vizeu Barrozo \\ Universidade de São Paulo Faculdade de Filosofia, Letras e Ciências Humanas Departamento de Geografia, São \\ Paulo, SP, Brasil \\ lija@usp.br \\ Guilherme Augusto Verola Mataveli
Universidade de São Paulo Faculdade de Filosofia, Letras e Ciências Humanas Departamento de Geografia, São
Paulo, SP, Brasil
mataveli@usp.br
}

\begin{abstract}
RESUMO
O Aedes aegypti é um vetor de agentes de doenças que tem caráter epidêmico como dengue, febre amarela urbana, chikungunya e Zika. Algumas estratégias têm sido adotadas para conter a dispersão desse mosquito, entretanto, ainda não foi alcançado o resultado desejado. A identificação de condições meteorológicas como temperatura de superfície e precipitação pluviométrica em escalas finas, junto com outras ações, pode contribuir para o controle da proliferação do Aedes aegypti. Neste sentido, foi realizada uma análise temporal de sete anos do valor máximo e mínimo da temperatura de superfície e precipitação pluviométrica acumulada associado à presença de Aedes aegypti para cada distrito da cidade de São Paulo. Para abstração desses valores, foram correlacionados produtos de sensoriamento remoto com casos autóctones de dengue. Os limiares encontrados para todo o município mostraram 55\% de precisão, 35\% de erro e 10\% foram inconclusivos devido à falta de dados de satélite por cobertura de núvens. No entanto, quando analisados individualmente alguns distritos atingiram uma precisão superior a $70 \%$. Este estudo apresenta pela primeira vez estimativas de limiares de variáveis meteorológicas em escala fina associadas à presença deste mosquito e torna possível identificar potenciais áreas de risco para a proliferação do Aedes aegypti com uma semana de antecedência.
\end{abstract}

Palavras-chave: Temperatura de superfície. Aedes aegypti. Imagens de satélite. Indicador de risco. Arboviroses.

\section{SURFACE TEMPERATURE AND PLUVIOSITY THRESHOLDS AS A INDICATOR OF Aedes aegypti PROLIFERATION RISK IN THE CITY OF SÃO PAULO}

\begin{abstract}
Aedes aegypti is a vector of diseases of epidemic character in Brazil, such as dengue, urban yellow fever, chikungunya and Zika. Some strategies have been adopt to contain the dispersion this mosquito, however, not was yet achieve the desired result. The identification of meteorological conditions such as surface temperature and rainfall at fine scales, along with other actions, may contribute to the control of_Aedes aegypti_proliferation. In this sense, a seven - year temporal analysis of the maximum and minimum values of surface temperature and accumulated precipitation associated to the presence of Aedes aegypti, it was made for each district of the city. For the abstraction of these values, remote sensing products were correlated with autochthonous cases of dengue. Thresholds found for the entire municipality showed $55 \%$ accuracy, $35 \%$ error, and $10 \%$ were inconclusive due to lack of temperature data. However, when analyzed individually, some districts reached an accuracy greater than $70 \%$. This study presents for the first time estimates of thresholds fine-scale meteorological variables associated with the presence in this mosquito and makes it possible to identify potential risk areas for the proliferation of_Aedes aegypti_a week in advance.
\end{abstract}

Keywords: Surface temperature. Aedes aegypti. Satellite image. Risk indicator. Arboviruses.

$\begin{array}{lllll}\text { Caminhos de Geografia } & \text { Uberlândia - MG } & \text { v. 21, n. 73 } & \text { Mar/2020 } & \text { p. 356-367 }\end{array}$ Página 356




\section{INTRODUÇÃO}

O Aedes aegypti é vetor dos vírus da dengue, Zika, chikungunya e, febre amarela urbana, que recentemente ressurgiu no país. O Aedes aegypti foi encontrado em mais de cento e trinta países localizaos na África, América, Europa e na região Ásia-Pacífico (ONU, 2016). Em 2015 com o aumento da disseminação do vírus Zika em vinte e quatro países das Américas, a Organização Mundial de Saúde (OMS) declarou emergência de saúde pública de importância internacional (FERNANDES, 2018). A dengue é considerada endêmica em mais de cento e vinte e oito países, colocando em risco quase quatro bilhões de pessoas.

No Brasil as doenças que têm os agentes transmitidos pelo Aedes aegypti tiveram significativa redução entre 2017 e 2018, a incidência de dengue mostrou redução de 30\%, a chikungunya e a Zika apresentaram redução de $55 \%$ e $89 \%$, respectivamente (NIVALDO, 2018). No entanto, este autor também destaca que neste mesmo período existiam quase mil e quinhentos municípios brasileiros em situação de alerta e mais de quatrocentos em situação de risco de epidemia. Este ano, até dois de fevereiro, segundo Penido (2019), o número de casos prováveis de dengue teve $149 \%$ de aumento, passando de mil novecentos casos para mais de cinquenta mil casos prováveis.

O controle dessas arboviroses é um grande desafio, pois para algumas anda não existem vacinas ou tratamentos específicos. Deste modo, é de extrema importância pesquisas voltadas para o desenvolvimento de produtos, técnicas e invenções que sejam mais eficazes para o controle do vetor e, consequentemente, das doenças por ele transmitidas (TEIXEIRA, 2008; NOGUEIRA e GUIMARÃES, 2015). Vasconscelos (2015), ressalta que a prevenção e o controle do mosquito transmissor são a melhor alternativa para combater estas doenças.

Tauil (2002) destaca que a prevenção é o melhor caminho e isso exige uma resposta rápida tendo a informação como ponto de partida para desencadear ações de controle. Tanto a informação como as ações tornam-se mais efetivas quando as áreas geográficas abordadas são mais restritas. Assim, buscar técnicas para desenvolver mapeamento de áreas de risco da presença deste vetor e integrar a todas as outras estratégias é imprescindível para fornecer sustentabilidade às ações estabelecidas pelas redes de vigilância (ZARA et al., 2016).

As características que definem as particularidades de cada local, como densidade da vegetação, densidade demográfica, tipo de habitação, características socioeconômicas, entre outras, agravam a formação da ilha de calor urbana (LOMBARDO, 1985), que está potencialmente associada à proliferação do mosquito (ARAUJO et al., 2015). O Aedes aegypti é muito sensível a elementos climatológicos, principalmente a temperatura que tem impacto sobre o tamanho da população do mosquito, pois interfere no período de incubação, na duração do período larval e pupal e na longevidade dos mosquitos adultos (GOMES et al., 2012). Os autores também destacam que a relação com a precipitação pluviométrica depende das características locais, apesar de os locais de reprodução serem mantidos predominantemente água da chuva acumulada.

Vários estudos buscam correlacionar as variáveis ambientais e climatológicas com a presença do vetor transmissor das arboviroses. Honorio (2009) expõe que criadouros são frequentemente encontrados nas áreas urbanas, sobretudo as de alta densidade demográfica e de baixa cobertura vegetal. Resendes et al. (2010), destacam que o grau de imunidade da população, índice de infestação pelo vetor e hábitos/comportamento da comunidade fazem parte da multicausalidade. Lima et al. (2016) afirmam que o clima, aliado as questões socioeconômicas, contribui para a proliferação de doenças epidemiológicas. No entanto, no estudo realizado por Araujo et al. (2015), que analisaram a incidência de dengue na cidade de São Paulo entre 2010-2011 em termos de variáveis ambientais e socioeconômica, verificaram uma alta incidência de dengue em zonas com elevada TS tanto em áreas de favela como em áreas com maior status socioeconômico. Também que as áreas de alta densidade populacional em São Paulo apresentaram menor incidência que as áreas de densidade populacional reduzidas e que as áreas de baixa cobertura de vegetação apresentam maior temperatura e maiores taxas de incidência de dengue. Eles concluíram que a TS foi o fator mais influente para a transmissão da dengue e que as pequenas flutuações da temperatura diária induzem maiores cargas virais nos mosquitos, o que também pode contribuir para a distribuição e a incidência de casos de dengue.

Os dados de temperatura do ar e precipitação pluviométrica obtidos pelas estações meteorológicas são restritos, devido à sua cobertura espacial e à sua escassez em muitas regiões do Brasil (ARAUJO et al. 2015). A limitação do alcance das medidas as torna inadequadas para capturar o gradiente de temperatura e pluviosidade de áreas mais distantes, subestimando os efeitos da temperatura e não

$\begin{array}{lllll}\text { Caminhos de Geografia } & \text { Uberlândia - MG } & \text { v. 21, n. 73 } & \text { Mar/2020 } & \text { p. 356-367 }\end{array}$ Página 357


Limiares de temperatura de superfície e pluviosidade como indicador de risco de proliferação de Aedes aegypti no município de São Paulo
Suely Franco Siquera de Lima Ligia Vizeu Barrozo Guilherme Augusto Verola Mataveli

permitindo, devido ao seu caráter pontual, capturar a variabilidade espacial da precipitação pluvial gerando incertezas nos resultados. Assim, a utilização de dados estimados por satélites mostra-se um importante recurso e são cada vez mais usados por permite a observação de qualquer região do planeta com várias resoluções espaciais (CAMPAROTTO et al., 2013; CHEN et al., 2018). Inclusive para monitorar a infestação de mosquitos vetores (Neteler et al 2011). Como bem destacado por Chen et al. (2018), as tecnologias geoespaciais emergentes são ferramentas poderosas para estimativas em escala mais detalhadas e, os conjuntos de dados amplamente disponíveis e a flexibilidade das técnicas de geoprocessamento, possibilitam o desenvolvimento de métodos eficazes e de baixo custo.

Assim sendo, este artigo apresenta os limiares de TS e precipitação pluviométrica acumulada (PA) que caracterizam o risco de proliferação do mosquito Aedes aegypti, obtidos de dados estimados por satélites, em escala distrital na cidade de São Paulo. Cabe ressaltar que estes resultados são inéditos e oriundos da complementação da metodologia apresentada em Lima et al. (2018). O estudo contribui para os contextos científicos, técnicos e administrativos que podem embasar as políticas públicas na prevenção, monitoramento e controle do Aedes aegypti.

\section{METODOLOGIA}

\section{Área de estudo}

O município de São Paulo, capital do estado de São Paulo, está localizado na região sudeste do Brasil e apresenta uma área total de $1.521 .110 \mathrm{~km}^{2}, 11.253 .503$ habitantes e densidade populacional de 7.398,26 habitante $/ \mathrm{km}^{2}$ (Instituto Brasileiro de Geografia e Estatística (IBGE), 2017). A cidade tem clima subtropical, com estação chuvosa concentrada nos meses de verão e outono (dezembro a maio) e estação seca nos meses de inverno e primavera (junho a novembro) (ARAUJO et al., 2015). Segundo o boletim climatológico publicado pelo Instituto Nacional de Meteorologia do Brasil (INMET) (2018), a precipitação anual normal de 1981-2010 para São Paulo foi de $1.616 \mathrm{~mm} / \mathrm{ano}$, e as faixas de temperatura anual mínima e máxima variaram de $16,2^{\circ} \mathrm{C}$ a $25,7^{\circ} \mathrm{C}$. São Paulo é subdividido em 96 distritos, como mostra a Figura 1.

Figura 1 - Município de São Paulo e seus distritos.

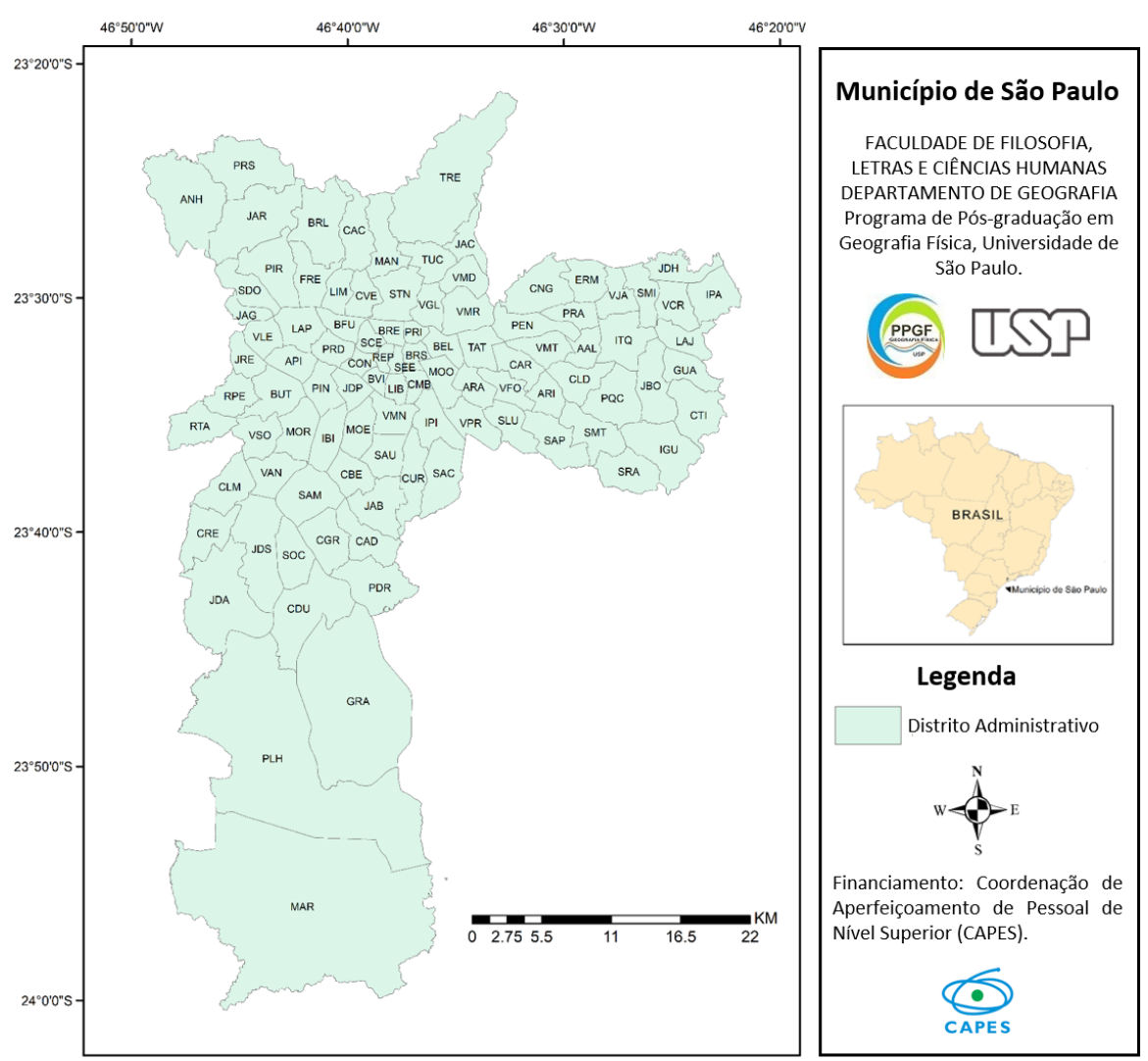

Fonte - Própria (2019). 
Limiares de temperatura de superfície e pluviosidade como indicador de risco de proliferação de Aedes aegypti no município de São Paulo
Suely Franco Siquera de Lima Ligia Vizeu Barrozo Guilherme Augusto Verola Mataveli

Cabe ressaltar que parte da metodologia aqui apresentada já foi descrita em Lima et al. (2018). Contudo, este artigo traz a complementação do trabalho e apresenta resultados mais avançados.

\section{Aquisição de dados e pré-processamento}

Esta etapa, já descrita na metodologia de Lima et al. (2018), consistiu na aquisição dos dados de TS do produto MOD11A1 (resolução espacial de $1 \mathrm{~km}$, resolução temporal de 1 dia e obtida às 13h30), provenientes do sensor MODIS a bordo do satélite Terra. O produto foi obtido do site USGS Earth Explorer - Pesquisa Geológica dos EUA, tile H13V11 que possue áreas de dez a dez graus em que está inserido o município de São Paulo (WAN et al., 2015). Após a aquisição, esses dados foram convertidos do Formato de Dados Hierárquicos (HDF) para TIF (Tagged Image File Format) e como estão em projeção sinusoidal (referência WGS84) foram reprojetadas para o sistema de coordenadas geográficas com o mesmo datum. Este pré-processamento foi realizado no software MODIS Reprojection Tool (MRT). No ArcGis, os valores de temperatura da superfície foram convertidos de Kelvin para Graus Celsius. Também descrita na metodologia de Lima et al. (2018), os dados da Missão de Medição de Precipitação Tropical (TRMM), produto 3B42, foram baixados gratuitamente no site da NASA. Este produto tem resolução temporal diária e espacial de $28 \mathrm{~km}$. Eles foram convertidos do formato NetCDF4 para o formato TIF e para a projeção WGS84, por meio de um script IDL (Interactive Data Language). Os dados epidemiológicos referentes ao número de casos autóctones de dengue de 2010 a 2016, organizados em SE e por distrito do município de São Paulo, foram adquiridos junto a Coordenadora da Coordenadoria de Vigilância em Saúde (COVISA) no formato de planilha Excel.

Para esse estudo foram obtidas cinco mil, cento e quatorze imagens. Após a aquisição e préprocessamento dos dados MODIS e do TRMM, foi calculado a moda da TS e da pluviosidade de cada distrito do município de São Paulo, ou seja, no ArcGis foi abstraído a moda do conjunto de pixel (imagem MODIS) que se encontra dentro do polígono que representa cada Distrito. Dado o volume de imagens, foi gerado um script (Python) para automatizar a análise. Antes de calcular a moda de precipitação pluviométrica, foi criada uma máscara para dividir o píxel do TRMM em $1 \mathrm{~km} X 1 \mathrm{~km}$, no mesmo tamanho do píxel dos dados do MODIS. Isso porque em alguns distritos havia parte de dois ou três pixels das imagens TRMM em diferentes proporções. As modas obtidas foram transferidas para planilha Excel que foram organizadas por ano de estudo (2010 - 2016) e em cada ano continha os 96 distritos do município de São Paulo e cada distrito com as 52 SE. A partir do primeiro dia de cada SE foram retroagidos 36 dias para que as variáveis ambientais coincidissem com a provável presença do mosquito que causaram a infecção dos casos notificados. O tempo de desenvolvimento do Aedes aegypti (as fases de ovo-larvaadulto) ocorre em um período que varia entre 5 e 7 dias; o período de incubação do vírus da doença (Dengue) no mosquito, varia entre 8 a 12 dias; o período de incubação do vírus no organismo do homem, pode variar de 2 a 10 dias; e o período para o indivíduo procurar o médico, realizar o exame e ocorrer a notificação, leva em média 7 dias (FIOCRUZ, 2019). Assim, considerando o prazo mínimo e máximo de todo o processo, levam de 22 e 36 a depender de as condições ambientais serem mais ou menos favoráveis ao mosquito e ao vírus (BRASIL, 2002; BESERRA et al. 2009; VALLE, 2013). Considerou-se o período entre o vigésimo segundo e o trigésimo sexto dia para estimar a mediana da moda da TS e o acumulado da moda da precipitação (LIMA e BARROZO, 2018).

A mediana da TS e precipitação acumulada (PA) foram correlacionadas com o número de casos relatados de dengue da SE correspondente (LIMA et al., 2018). Os gráficos gerados possibilitaram identificar a TS e - AP que ocorreram nos dias em que possivelmente estavam presentes os mosquitos que transmitiram o vírus e que resultaram nos casos notificados de cada SE. Também identificar quais foram o AP e a TS favoráveis e desfavoráveis a proliferação do mosquito para cada distrito em cada ano.

\section{Cálculo dos limiares de TS e AP correlacionados com a proliferação de Aedes aegypti}

Com base nos gráficos gerados, foi identificado para todos os anos estudados (2010 a 2016) e para cada distrito, quais foram as TS e AP iniciais, isto é, aquelas que coincidem com as primeiras SE que apresentaram casos notificados; TS e AP finais, aquelas que coincidem com as últimas SE que

\begin{tabular}{|c|}
\hline Vaminhos de Geografia \\
\hline
\end{tabular}


Limiares de temperatura de superfície e pluviosidade como indicador de risco de proliferação de Aedes aegypti no município de São Paulo

apresentaram casos notificados; TS e AP ideais, que coincidem com as SE que apresentaram maior número de casos notificados. Após, com esses valores, foram feitas análises combinatória e de probabilidade e concluiu-se que os AP ideais e as TS iniciais, máximas e mínimas observadas nos sete anos de estudo, foram os valores que mostraram maior correlação com a presença dos mosquitos. Deste modo, adotou-se esses valores como os limiares procurados.

Para avaliar se os limiares encontrados são representativos, foi analisado para cada dia de cada ano de estudado se os dias que apresentaram a TS e o AP dentro dos parâmetros dos limiares, culminaram em casos notificados de dengue. Neste processo, pelo mesmo motivo que foram retroagidos os dias a partir do início de cada SE, os dias foram adiantados a partir da data em que foram detectados os limiares e analisado se as SE que se encontravam entre o vigésimo segundo e trigésimo sexto dias avançados apresentavam casos de dengue.

\section{RESULTADOS}

\section{Cálculo da moda da TS e da pluviosidade}

No ano de 2016 em todo o município, a moda da pluviosidade constatou valores de mínimo e máximo entre 0 e $118 \mathrm{~mm}$, muito próximo da média histórica de $188,4 \mathrm{~mm}$ divulgada pelo Instituto Nacional de Meteorologia (INMET, 2018). Isso mostra a viabilidade dos dados de satélites.

A moda da TS máxima observada nos anos de 2010, 2011, 2012 e 2013 foram respectivamente de $43^{\circ} \mathrm{C}$, $44^{\circ} \mathrm{C}, 44^{\circ} \mathrm{C}$ e $43^{\circ} \mathrm{C}$ e chegaram a $45^{\circ} \mathrm{C}$ nos anos de 2014,2015 e 2016. INMET (2018) divulga que média normal das mínimas e máximas temperatura do ar ocorridas em março, entre 1981 a 2010 foram de $18^{\circ} \mathrm{C}$ e $28^{\circ} \mathrm{C}$ respectivamente e as ocorridas em março 2018 foram de $20,3^{\circ} \mathrm{C}$ e $29,5^{\circ} \mathrm{C}$, evidenciando o mesmo aumento de aproximadamente $1^{\circ}$ de temperatura depois de 2010. Cabe ressaltar que nestes últimos três anos ocorreu um aumento no número de casos de dengue exponencial, passando de 2.610 casos em 2014 para 100.681 casos em 2015.

\section{Cálculo da mediana da TS e do AP}

O cálculo da mediana da TS e do acumulado pluviométrico foi necessário para se estabelecer um valor único de temperatura e pluviosidade que representasse o intervalo de tempo associado a cada SE. Não se sabe ao certo quantos $\mathrm{mm}$ de chuva são necessários para criar ambientes propícios para os criadouros dada às singularidades de cada lugar, mas se sabe que é necessário um período chuvoso e com limitações para criar condições para a proliferação do mosquito (PEREDA, 2011).

\section{Abstração dos limiares de temperatura de superfície e pluviosidade}

Observou-se que a presença do Aedes Aegypti em função dos casos notificados de dengue, em cada distrito, ocorria entre os valores mínimo e máximo das TS iniciais e do AP ideais. Assim, estes valores foram considerados como mais representativos para serem adotados como limiares. Deste modo, para cada distrito, entre todos os anos estudados, foi abstraído o valor mínimo e máximo da TS inicial e da pluviosidade ideal como sendo o limiar (Figuras 2 e 3 ). 
Figura 2 - Limiares de temperatura da superfície $\left({ }^{\circ} \mathrm{C}\right)$ favoráveis à proliferação de Aedes aegyptị para cada distrito da cidade de São Paulo.

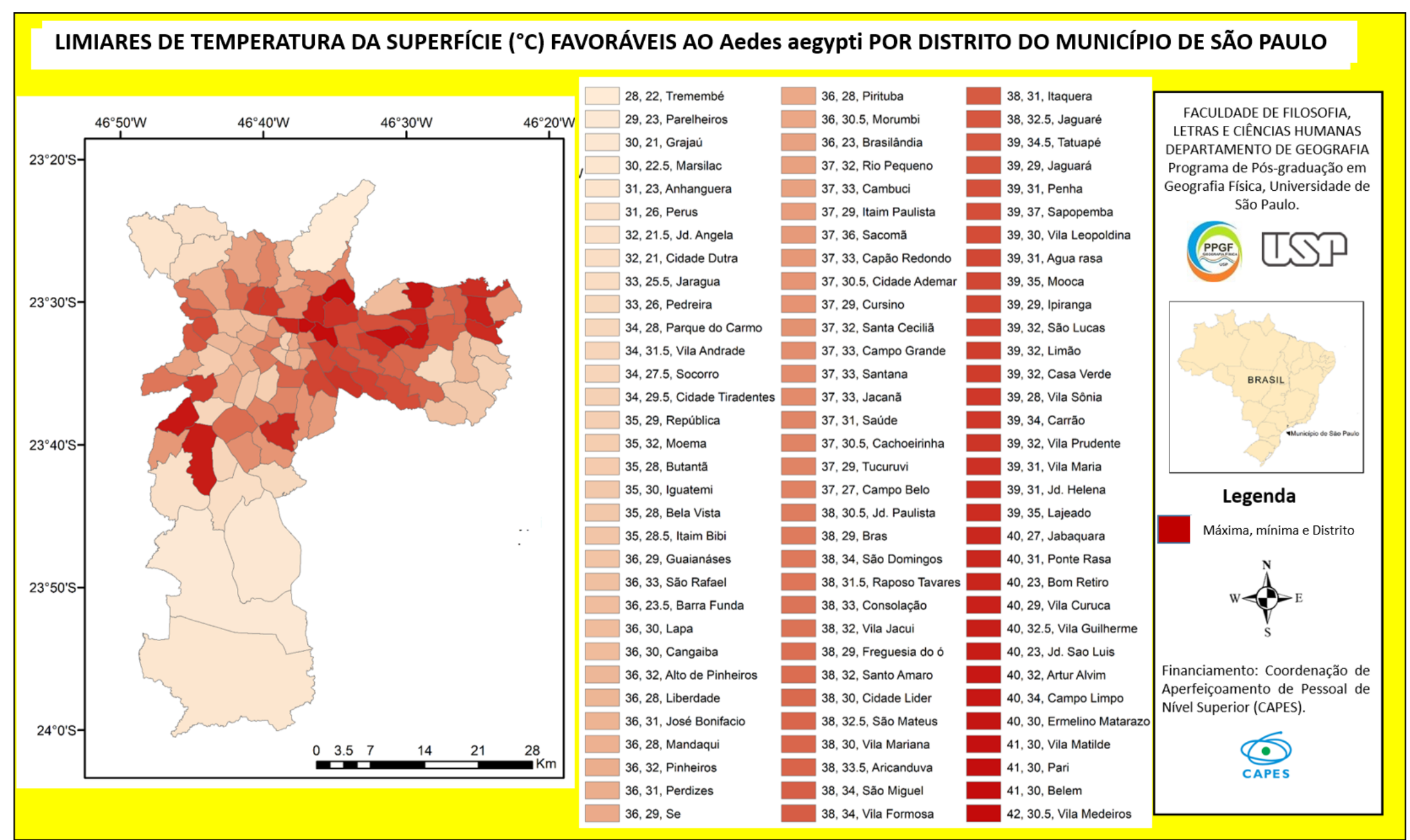

Fonte - Própria (2019). 
Figure 3 - Limiares de acumulado pluviométrico $(\mathrm{mm})$ favoráveis à proliferação de Aedes aegypti para cada distrito da cidade de São Paulo.

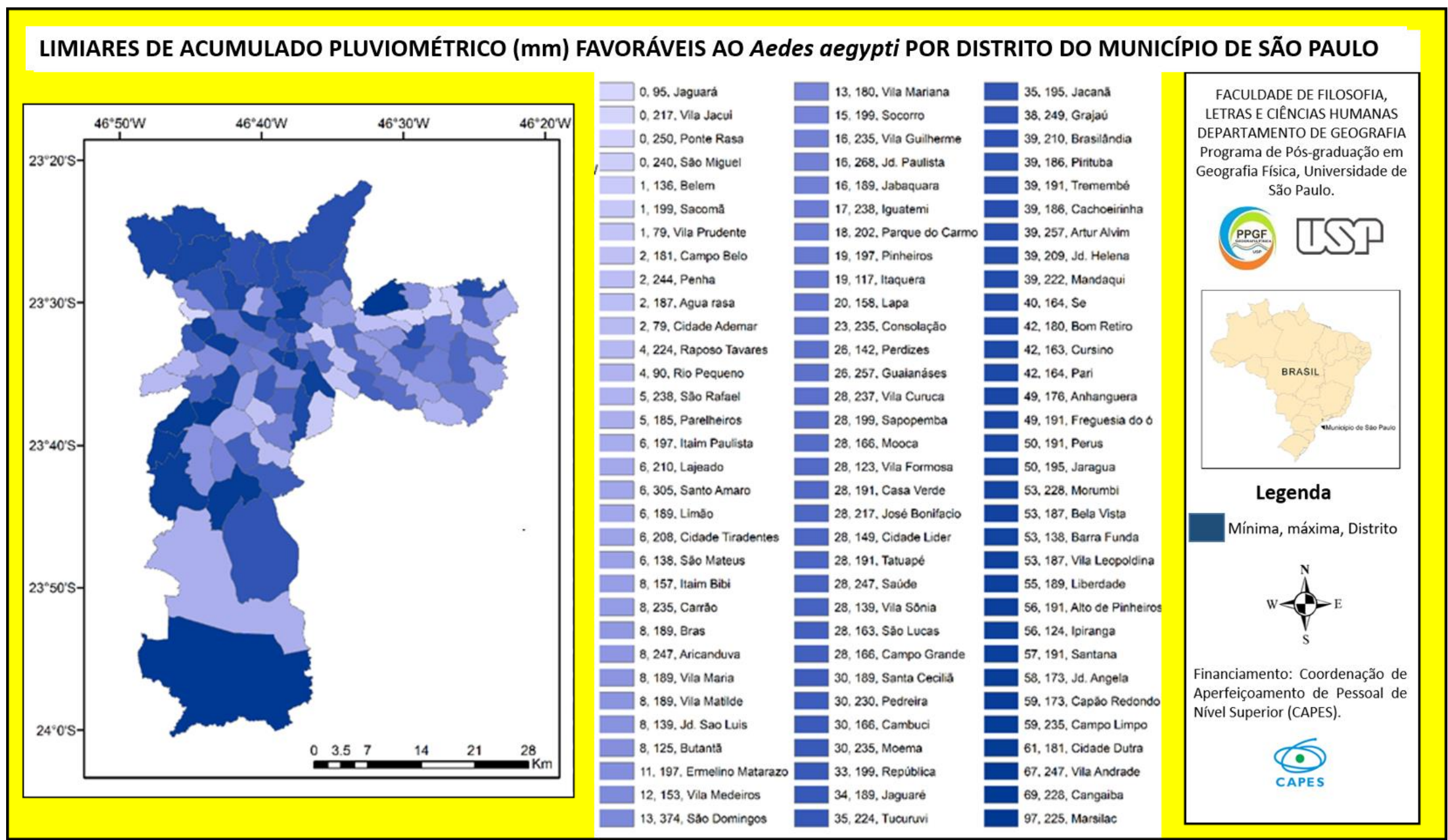

Fonte - Própria (2019). 


\section{Avaliação da representatividade dos limiares encontrados}

Para a avaliação dos limiares foram observados todos os dias de cada ano de estudo a TS e o AP de cada distrito. O objetivo foi averiguar se quando os valores dos elementos climáticos se enquadravam nos valores dos limiares, havia a notificação de casos de dengue, para isso, três estratégias foram adotadas. A primeira foi considerar um acumulado pluviométrico dos sete dias anteriores a data em que se constatou o limiar da TS. Por exemplo, se no dia 8 de março constatou-se, para o Distrito Vila Sônia, uma temperatura dentro do parâmetro do limiar, entre 28 e $39^{\circ} \mathrm{C}$, soma-se os milímetros de chuva dos dias 08/03, 07/03, $06 / 03,05 / 03,04 / 03,03 / 03,02 / 03$. Se o valor encontrado estiver de acordo com o limiar proposto para este distrito, entre 28 e $139 \mathrm{~mm}$, considera-se este dia (08/03) como propício para o desenvolvimento do Aedes aegypti. Mesmo que não chova no dia em que a temperatura atingiu valores do limiar, pode ter chovido alguns dias antes, abastecendo os criadouros. Também, como destacado por Pereda et al. (2011), é necessário um período chuvoso para criar condições para o crescimento do mosquito. A segunda é avançar os 36 dias a partir do dia 08/03 e verificar se no intervalo entre o vigésimo segundo dia e trigésimo sexto há SE com casos notificados de dengue. Os dias são avançados para considerar o período de desenvolvimento do mosquito, incubação do vírus e notificação da doença. A Terceira foi classificar os dias como alarme: 1) FALSO, quando ocorre os valores dos limiares e não há notificação de casos; 2) CORRETO, quando ocorre os valores dos limiares e há notificação de casos; 3) NÃO FUNCIONOU, quando não ocorre os valores dos limiares e há notificação de casos; 4) NÃO HOUVE, quando não ocorre os valores dos limiares e não há notificação de casos; 5) INCONCLUSIVO, quando não há valores de TS em consequência da presença de nuvens. Como quando avançados os dias ( $2^{\mathrm{a}}$ estratégia) é analisado entre o vigésimo segundo dia e trigésimo sexto dia, ocorre de abranger três SE neste período, fazendo-se necessário criar uma lógica de prioridades: 1- ALARME FALSO prevalece sobre: Inconclusivo / Não funcionou /Não houve alarme / Correto. 2-ALARME CORRETO prevalece sobre: Inconclusivo / Não funcionou / Não houve alarme. 3- ALARME NÃO FUNCIONOU prevalece sobre: Inconclusivo / Não houve alarme. 4-NÃO HOUVE ALARME prevalece sobre: Inconclusivo. 5-ALARME INCONCLUSIVO não prevalece sobre nenhum / só vai aparecer inconclusivo quando houver só ele.

Foram analizados dois mil, quinhentos e cinquenta e sete dias entre 2010 e 2016 para cada distrito. Conclui-se que no geral, isto é, analisando todos os distritos juntos, $55 \%$ dos dias analisados os limiares funcionaram (Alarme Correto e Não Houve Alarme) e falharam em $35 \%$ dos dias (Alarme Falso e Alarme Não Funcionou). Porem, quando analisado os distritos individualmente, observa-se resultados mais favoráveis que o geral, como exemplo o distrito Alto de Pinheiros, que em $82.7 \%$ dos dias analisados os limiares funcionaram (Tabela 1).

Tabela 1 - Avaliação dos limiares de Temperatura de Superfície e Acumulado Pluviométrico do distrito Alto de Pinheiros do município de São Paulo - SP no ano de 2012.

\begin{tabular}{|lcc|}
\hline \multicolumn{3}{c|}{ Teste } \\
Bairro & ALTO DE PINHEIROS \\
ANO & $\mathbf{2 0 1 2}$ & \\
\hline Rótulos & Contagem de dias & Contagem de dias em \% \\
\hline Alarme Correto & 14 & $3.83 \%$ \\
Alarme Falso & 42 & $11.48 \%$ \\
Alarme Inconclusivo & 14 & $3.83 \%$ \\
Alarme Não Funcionou & 7 & $1.91 \%$ \\
Não Houve Alarme & 289 & $78.96 \%$ \\
\hline Total Geral & $\mathbf{3 6 6}$ & $\mathbf{1 0 0 . 0 0 \%}$ \\
& Fonte - Própria (2019). \\
\hline
\end{tabular}

\section{DISCUSSÃO}

Estudos prévios mostraram a necessidade de pesquisas com foco no desenvolvimento de métodos, produtos e técnicas eficazes para o controle do Aedes aegypti e das doenças cujos agentes são transmitidos por eles. E que a melhor alternativa para o controle do mosquito é a prevenção e ações direcionadas localmente (TEIXEIRA, 2008; TAUIL, 2002). Outras pesquisas esclareceram que as diferenças de temperatura e precipitação são fatores de alta relevância no crescimento da população do

$\begin{array}{llllll}\text { Caminhos de Geografia } & \text { Uberlândia - MG } & \text { v. 21, n. 73 } & \text { Mar/2020 } & \text { p. 356-367 } & \text { Página } 363\end{array}$


Aedes aegypti e enfatizaram a necessidade de estudos em escalas locais em áreas urbanas (GOMES et al., 2012; ARAUJO et al., 2015). Em geral, os estudos da influência da temperatura nesses mosquitos são realizados em escalas laboratoriais, municipais, estaduais, regionais e até globais, mas não em escalas mais sutis dentro da região urbana. Deste modo, este estudo considerou tanto a vulnerabilidade do Aedes aegypti à temperatura da superfície e a precipitação pluviométrica, como também a falta de informação dessas variáveis em escalas mais pontuais, necessárias para embasar as ações dos agentes de saúde.

Com base em estudos anteriores sobre a aquisição dessas variáveis por meio de geotecnologias, foram integrados produtos de sensoriamento remoto livre com dados de saúde para a aquisição dos limiares de TS e PA em escalas locais. O método permite gerar mapas de risco da presença do mosquito em escala de até $1 \mathrm{~km}$ para qualquer região do planeta.

Os resultados preliminares mostram que, no geral, os limiares funcionaram em $55 \%$ dos dois mil, quinhentos e cinquenta e sete dias analisados, mas também que alguns distritos atingiram mais de $80 \%$ de precisão. Estes resultados podem ainda ser melhorados, pois os valores foram obtidos automaticamente e não passaram por uma revisão de uma pessoa especialista, o que traria maior precisão aos limiares. A amplitude entre os limites máximo e mínimo é exagerada e pode ser ajustada com a análise de um especialista.

Observou-se que quando analisados apenas o período sazonal de incidência da doença, os resultados são mais satisfatórios, possivelmente por se tratar de um período que coincide com maior ocorrência de chuvas e aumento da temperatura superficial. No entanto, a doença também ocorreu em menor escala durante o período fora da sazonalidade, demonstrando que é necessário monitoramento constante. A doença pode ocorrer em qualquer local e período do ano, quando há condições ambientais favoráveis a presença do vetor e população humana suscetível.

Outro ponto de relevância para o aprimoramento e que não foi possível para este trabalho, seria usar como dado de saúde, ao invés de casos notificados de dengue o Índice de Breteau e/ ou Indice Predial. Este tipo de Indicador tem sido usado na avaliação da estimativa da densidade larvária do Aedes aegypti. É um cálculo feito a partir de inspeção de uma amostra probabilística do total de imóveis da área. É a relação entre o número de recipientes positivos e o número de imóveis pesquisados na área (BRASIL 2005). Este dado de saúde possibilita identificar com mais precisão o local e o momento em que o mosquito se faz presente. Isso eliminaria algumas etapas da metodologia, reduzindo a margem de erro na abstração dos limiares, melhorando o percentual de acerto e diminuindo o percentual de resultados falsos. Cabe ressaltar que existem problemas que interferem na conscistencia dos dados sobre casos notificados de dengue, como destacado nos estudos realizados por Toledo et al. (2006); Duarte e França (2006); Barbosa et al. (2015); Silva et al. (2018), entre outros.

Também é possível melhorar a precisão usando outros produtos de sensoriamento remoto. $O$ produto MOD11A1 é muito eficiente porque é fornecido com resolução espacial de $1 \mathrm{~km}$ e resolução temporal de um dia, por outro lado, o produto 3B42 com resolução espacial de $28 \mathrm{~km}$, pode ser substituído, por exemplo, pelo produto Climate Hazards group InfraRed Precipitation with Station Data (CHIRPS), que conciste em dados diários de precipitação com resolução espacial de $5 \mathrm{~km}$ e também são disponibilizados gratuitamente com datas a partir de 1981 (Funk et al., 2015).

Para uma melhor avaliação dos limiares são necessários estudos com dados de sensoriamento remoto e de saúde, em tempo real, dos anos subsequentes. No entanto, o método demonstrou ser viável, já que permite identificar variáveis de influência na reprodução do mosquito em escala sutil e gerar dados para compor um mapa de risco com dias de antecedência para orientar o trabalho dos agentes de saúde e a população.

A escala distrital utilizada neste trabalho, é viável para parametrizar sistemas de alerta para a população, que também desempenham um papel importante no combate desse vetor. Para orientar as redes de vigilância locais para o desenvolvimento de um planejamento mais eficiente com mitigação de custo e tempo, escalas maiores são necessárias, o que também é possível a partir dessa metodologia. Dessa forma, compartilhar este estudo é importante e em especial para os países em desenvolvimento que possuem recursos limitados nos setores de saúde pública e sofrem com as doenças transmitidas pelo Aedes aegypti.

\begin{tabular}{|c|}
\hline Jaminhos de Geografia \\
\hline
\end{tabular}




\section{CONSIDERAÇÕES FINAIS}

Este estudo demonstra que é possível fornecer uma nova abordagem para a vigilância epidemiológica, explicada pela distribuição dos valores das variáveis climatológicas de temperatura superficial e precipitação. A principal contribuição é a identificação da área de risco à proliferação do Aedes aegypti em escalas finas e com a possibilidade de ações preventivas e não corretivas, como vem sendo praticado no Brasil. Além disso, apresenta uma metodologia viável para qualquer cidade do país ou mesmo para outros países, por usar dados de sensoriamento remoto.

Com as possibilidades de melhorias já identificadas na metodologia e com as sugestões propostas para alcançar resultados mais eficazes, espera-se que ela seja aprimorada, usada e contribua para potencializar a eficácia das abordagens de vigilância epidemiológica existentes, culminando em maior controle da população deste mosquito e na mitigação dos poucos recursos disponíveis em saúde pública.

\section{AGRADECIMENTOS}

Expressamos nosso agradecimento a CAPES pela bolsa de pós-doutorado concedida para a realização da pesquisa que embasou a redação desse artigo e a COVISA pelo apoio no compartilhamento dos dados epidemiológicos.

\section{REFERENCES}

ARAUJO, R.V.; ALBERTINI, M.R.; SILVA, A.L.C.; SUESDEK, L.; FRANCESCHI, N.C.S.; BASTOS, N.M.; KATZ, G.; CARDOSO, V.A.; CASTRO, B.C.; CAPURRO, M.L.; ALLEGRO, V.L.A.C. São Paulo urban heat islands have a higher incidence of dengue than other urban areas. The Brazilian Journal of Infectious Diseases. Salvador - BA, v.1 9, n.2, p. 146-155, 2015. doi.org/10.1016/j.bjid.2014.10.004.https://doi.org/10.1016/j.bjid.2014.10.004

BARBOSA, J. R.; BARRADO, J. C. S.; ZARA, A. L. S. A.; SIQUEIRA JÚNIOR, J. B. Avaliação da qualidade dos dados, valor preditivo positivo, oportunidade e representatividade do sistema de vigilância epidemiológica da dengue no Brasil, 2005 a 2009. Epidemiol. Serv. Saúde, Brasília, n. 24, v. 1, p: 49-58, 2015.https://doi.org/10.5123/S1679-49742015000100006

BESERRA, E.B.; FERNANDES C.R.M.; SILVA L.A.; SANTOS, J.W. Efeitos da temperatura no ciclo de vida, exigências térmicas e estimativas do número de gerações anuais de Aedes aegypti (Diptera, Culicidae). Iheringia, Sér. Zool, v.2, n.99, p. 142-148, 2009.https://doi.org/10.1590/S0073$\underline{47212009000200004}$

BRASIL. Ministério da Saúde. Fundação Nacional de Saúde. Série A. Normas e Manuais Técnicos. Dengue: aspectos epidemiológicos, diagnóstico e tratamento. (Série A. Normas e Manuais Técnicos, no 176) Ed. Ministério da Saúde, Brasília, DF. 2002. 20p.

BRASIL. Ministério da Saúde. Secretaria de Vigilância em Saúde. Diretoria Técnica de Gestão. Diagnóstico rápido nos municípios para vigilância entomológica do Aedes aegypti no Brasil - LIRAa: metodologia para avaliação dos índices de Breteau e Predial. (Série A. Normas e Manuais Técnicos) Ed. Ministério da Saúde, Brasília, DF. 2005, 60p. ISBN 5-334-1032-8.

CAMPAROTTO, L.B.; BLAIN, G.C.; GIAROLLA, A.G.C.A.; ADAMI, M.; CAMARGO, M.B.P. Validação de dados termopluviométricos obtidos via sensoriamento remoto para o Estado de São Paulo.

Revista Brasileira de Engenharia Agrícola e Ambiental. Campina Grande - PB, v.17, n. 6, p. 665-671, 2013. Doi: $10.1590 / S 1415-43662013000600013$.https://doi.org/10.1590/S1415-43662013000600013

CHEN, Q.; DING M.; YANG, X.; HU K.; QI, J. Spatially explicit assessment of heat health risk by using multi-sensor remote sensing images and socioeconomic data in Yangtze River Delta, China. International Journal of Health Geographics. V.17, n.15, p. 1-15, 2018. Doi: 10.1186 / s12942-0180135-y.https://doi.org/10.1186/s12942-018-0135-y

DUARTE, H. H.; FRANÇA, E. B. Qualidade dos dados da vigilância epidemiológica da dengue em Belo Horizonte, MG. Rev Saúde Pública, n.40, v. 1, p:42-134, 2006.https://doi.org/10.1590/S0034-

$\begin{array}{llllll}\text { Caminhos de Geografia } & \text { Uberlândia - MG } & \text { v. 21, n. 73 } & \text { Mar/2020 } & \text { p. 356-367 } & \text { Página } 365\end{array}$


Limiares de temperatura de superfície e pluviosidade como indicador de risco de proliferação de Aedes aegypti no município de São Paulo
Suely Franco Siquera de Lima Ligia Vizeu Barrozo Guilherme Augusto Verola Mataveli

\section{$\underline{89102006000100021}$}

FERNANDES, V.R.; MONKEN M.; GONDIN, G.M.M.; LUZ, Z.M.P.; LOPES, A.B.A.S.; CASTRO, M.C.; FILHO CASTRO, E.; LIMA, A.L.S.; SILVA, J.P.V.; AMORIM, A.C. Denaturalizing "Long-Lasting Endemic Diseases": Social Mobilization in the Context of Arboviral Diseases in Brazil. In: Globalization and Health Inequities in Latin America. Malagón, S. L., Luján V. R. Globalization and Health Inequities in Latin America. Springer Cham, 2018, 91-106, ISBN: 978-3-319-67292-2. https://doi.org/10.1007/978-3-319-67292-2 5

FIOCRUZ. Instituto Osvaldo Cruz - Aedes aegypti: vírus e vetor. Disponível em: <http://www. http://www.ioc.fiocruz.br/dengue/index.html>. Acesso em: 02 de set. 2019.

FUNK C.; PETERSON P.; LANDSFELD M.; PEDREROS D.; VERDIN J.; SHUKLA S.; GREGORY H.G.; ROWLAND, J.; HARRISON, L.; HOELL, A.; MICHAELSEN, J. The climate hazards infrared precipitation with stations-a new environmental record for monitoring extremes. Scientific data, 2015, 1 - 21p. Doi: 10.1038/sdata.2015.66.https://doi.org/10.1038/sdata.2015.66

GOMES, A.F.; NOBRE, A.A.; CRUZ, O.G. Análise temporal da relação entre dengue e variáveis meteorológicas na cidade do Rio de Janeiro, Brasil, 2001-2009. Cad. Saúde Pública. v.28 n. 11, p.2189-2197, 2012. Doi.org/10.1590/S0102-311X2012001100018.https://doi.org/10.1590/S0102$\underline{311 \times 2012001100018}$

HONORIO, N.A. Indicadores da distribuição espacial e temporal de Aedes (Stegomyia) aegypti (Linnaeus, 1762) (Diptera: Culicidae) associados às variáveis climáticas, ambientais e transmissão de dengue. Tese (Doutorado), Instituto Oswaldo Cruz - FIOCRUZ. 2009. Disponível em: <https://www.arca.fiocruz.br/handle/icict/5826>. Acesso em: 02 de set. 2019.

INMET - Instituto Nacional de Meteorologia. Boletim climatológico do Estado de São Paulo: março de 2018. Disponível em:

<http://www.inmet.gov.br/portal/notas_tecnicas/2018/ABRIL/Boletim_climaSP_MARCO_2018.pdf>. Acesso em: 09 de Julh. de 2018.

IBGE - Instituto Brasileiro de Geografia e Estatística (2017). Base de dados por municípios das Regiões Geográficas Imediatas e Intermediárias do Brasil. Disponível em:

<htpp://cidades.ibge.gov.br/brasil/sp/sao-paulo/panorama>. Acesso em: 09 Julh. de 2018.

LIMA, R.C.; MOREIRA, E.B.M.; NÓBREGA, R.S. A influência climática sobre a epidemia dengue na cidade do Recife por Sistema de Informações Geográficas. Revista Brasileira de Geografia Física. v.9, n.2, p.384-398, 2016. ISSN: 1984-2295 https://doi.org/10.5935/1984-2295.20160027

LIMA, S.F.S.; BARROZO, L.V. Produto Surface Temperature and Precipitation that Influence the Incidence of Aedes Aegypti in São Paulo. Revista do Departamento de Geografia - Volume Especial, p.174-182, 2018. DOI: 10.11606/rdg.v0ispe.145697. https://doi.org/10.11606/rdg.v0ispe. 145697

NETELER, M.; ROIZ, D.; ROCCHINI, D.; CASTELLANI, C.; RIZZOLI A. Terra and Aqua satellites track tiger mosquito invasion: modelling the potential distribution of Aedes albopictus in north-eastern Italy. International Journal of Health Geographics. 2011. https://doi.org/10.1186/1476-072X-10-49 https://doi.org/10.1186/1476-072X-10-49

LOMBARDO, M. A. Ilha de Calor nas Metrópoles: o exemplo de São Paulo. São Paulo: HUCITEC, 1985.

NIVALDO, C. Agência Saúde. Ministério da Saúde. Ministério da Saúde mobiliza população para o combate ao Aedes aegypti. Disponível em: <http://portalms.saude.gov.br/noticias/agenciasaude/42656-ministerio-da-saude-mobiliza-populacao-para-o-combate-ao-aedes-aegypti>. Acessado em: 18 de Jun. de 2018.

NOGUEIRA, P.; GUIMARÃES, M. Um vilão de muitas caras. Revista Pesquisa FAPESP. 2015, 16 23p. 
ONU - Organização das Nações Unidas - News (2016). Organização Mundial da Saúde alerta para um dos "seres" mais mortais do mundo. Disponível em:

$<$ https://news.un.org/pt/story/2016/08/1559761-oms-alerta-para-um-dos-seres-mais-mortais-domundo>. Acessado em: 4 de Jul de 2018.

PEREDA, P.C.; ALVES, D.C.O.; RANGEL, M.A. Elementos Climáticos e Incidência de Dengue: Teoria e Evidência para Municípios Brasileiros. 2011. Disponível em:

<http://bibliotecadigital.fgv.br/ocs/index.php/sbe/EBE11/paper/download/2938/1333>. Acessado em: 08 d Agost de 2016.

PENIDO, A. Ministério da Saúde alerta para aumento de 149\% dos casos de dengue no país. Disponível em: <http://portalms.saude.gov.br/noticias/agencia-saude/45257-ministerio-da-saudealerta-para-aumento-de-149-dos-casos-de-dengue-no-pais>. Acessado em: 21de março de 2019.

RESENDES, A.P.C.; SILVEIRA, A.P.R.; SABROZA, P.C.; SANTOS, R.S. Determinação de áreas prioritárias para ações de controle da dengue. Revista Saúde Pública, v. 44; n. 2, p. 1-8, 2010. https://doi.org/10.1590/S0034-89102010000200007

SILVA, N. M.; TEIXEIRA, R. A. G.; JUNIOR SIQUEIRA, J. B.; COELHO, G. E.; OLIVEIRA, E.S.F. Vigilancia de chikungunya en Brasil: desafíos en el contexto de la Salud Pública. Epidemiol. Serv. Saude, Brasília, v. 27, n. 3. e:2017127, 2018. doi: 10.5123/S1679-49742018000300003. https://doi.org/10.5123/S1679-49742018000300003

TAUIL, P.L. Critical aspect of dengue control in Brazil. Cad. Saúde Pública, Rio de Janeiro. V.18, n.3, p.867-871, 2002. Doi: 10.1590/S0102- 311X2002000300035. https://doi.org/10.1590/S0102$\underline{311 \times 2002000300030}$

TEIXEIRA, M.G. Controle do dengue: importância da articulação de conhecimentos transdisciplinares. Interface Comunicação Saúde Educação, v.12, n.25, p. 442-51, 2008. https://doi.org/10.1590/S1414-32832008000200019

TOLEDO, A. L. A.; ESCOSTEGUY, C. C.; MEDRONHO, R. A.; ANDRADE, F. C. Confiabilidade do diagnóstico final de dengue na epidemia 2001-2002 no Município do Rio de Janeiro, Brasil. Cad. Saúde Pública, v. 22, n. 5, p:933-940, 2006. https://doi.org/10.1590/S0102-311X2006000500006

[dataset] Tropical Rainfall Measuring Mission (TRMM) (2011), TRMM (TMPA) Rainfall Estimate L3 3 hour 0.25 degree x 0.25 degree V7, Greenbelt, MD, Goddard Earth Sciences Data and Information Services Center (GES DISC). Acessado em: 31 de Jan. de 2019. 10.5067/TRMM/TMPA/3H/7.

VALLE, D., coordenadora. Aedes aegypti - Introdução aos Aspectos Científicos do Vetor. Projeto vídeo-aulas Instituto Oswaldo Cruz (IOC/Fiocruz) [Vídeo-aulas], 2013. Rio de Janeiro: IOC Fiocruz. Disponível em: <http://auladengue.ioc.fiocruz.br/>. Acessado em: 10 de Agost. De 2018.

VASCONSELOS, P.F.C. Doença pelo vírus Zika: um novo problema emergente nas Américas? Revista Pan-Amazônica de Saúde. v.6, p.9-10, 2015. Doi: 10.5123/S2176-6223201500020000. https://doi.org/10.5123/S2176-62232015000200001

[Data set] WAN, Z.; HOOK, S.; HULLEY, G. (2015). MOD11A1 MODIS/Terra Land Surface Temperature/Emissivity Daily L3 Global $1 \mathrm{~km}$ SIN Grid V006. NASA EOSDIS LP DAAC. doi: 10.5067/MODIS/MOD11A1.006.

ZARA, A.L.S.A.; SANTOS, S.M.; OLIVEIRA, E.S.F.; CARVALHO, R.G.; COELHO, G.E. Estratégias de controle do Aedes aegypti: uma revisão. Brasília. Epidemiol. Serv. Saúde. v.25, n.2, p.391-404, 2016. Doi: $10.5123 / S 1679-49742016000200017$. https://doi.org/10.5123/S1679-49742016000200017

Recebido em: 17/05/2019

Aceito para publicação em: 17/06/2019

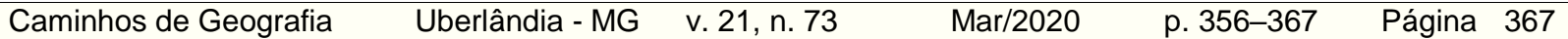

